\title{
Hemolytic profile of novel tri-heterocyclic benzamides
}

\begin{abstract}
Heterocyclic compounds containing five-membered or six-membered heterocyclic units have a diversity of valuable biological effects. In the present work, some novel tri-heterocyclic benzamides, $8 \mathrm{a}-\mathrm{g}$, were synthesized in multi-steps. The benzamide containing electrophile, $\{4$-[4-(chloromethyl)benzoyl]-1-piperazinyl $\}$ (2-furyl) methanone (3), was synthesized by the reaction of 4-(chloromethyl)benzoyl chloride (2) and 2-furoyl-(1-piperazinyl) methanone (1) in a basic aqueous medium. In parallel series of steps, substituted-benzoic acids (4a-g) were refluxed with ethanol and conc. sulfuric acid to form respective ethyl substituted-benzoates (5a-g). These esters were further refluxed with $\mathrm{N} 2 \mathrm{H} 4 . \mathrm{H} 2 \mathrm{O}$ in methanol solution to acquire substitutedbenzohydrazides $(6 \mathrm{a}-\mathrm{g})$. These hydrazides were cyclized into heterocyclic core by refluxing with $\mathrm{CS} 2$ in the presence of $\mathrm{KOH}$ and ethanol solvent, whereby yielding various 5-(substituted-phenyl)-1,3,4-oxadiazol-2-thiols (7a-g). In the final step, the electrophile 3, was refluxed with synthesized 1,3,4-oxadiazoles, 7a-g, in acetonitrile and potassium carbonate to acquire the targeted novel tri-heterocyclic benzamides, 8a-g. The structural characterization of these newly synthesized molecules was done by IR, 1H-NMR, 13C-NMR, and EI-MS spectral data. All these compounds were evaluated for their hemolytic activity to ascertain their cytotoxicity profile.
\end{abstract}

Keywords: novel tri-heterocycles, 1H-NMR, 13C-NMR, EI-MS, hemolytic activity, heterocyclic compounds
Volume 5 Issue 3 - 2018

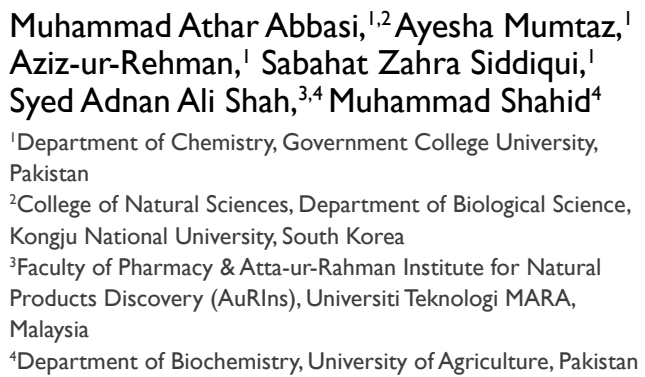

Correspondence: Muhammad Athar Abbasi, Department of Chemistry, Government College University, Lahore-54000, Pakistan, Tel (+92)-42-1 I I0000 I0, Ext 266, Email atrabbasi@yahoo.com, abbasi@gcu.edu.pk

Received: December 04, 2017 | Published: May 09, 2018
Abbreviations: OLED, organic light-emitting diodes; $\mathrm{KOH}$, potassium hydroxide; NMR, nuclear magnetic resonance; TLC, thin layer chromatography; EDTA, ethylene diamine tetra acetic acid; PBS, Phosphate-buffered saline; HEC, higher education commission; EI-MS, electron ionization mass spectrometry

\section{Introduction}

Oxadiazoles are the heterocyclic compounds containing one oxygen and two nitrogen atoms in a five membered ring, ${ }^{1}$ possessing a diversity of useful biological effects. ${ }^{2}$ Oxadiazole is considered to be resultant from furan by replacement of two methane $(-\mathrm{CH}=)$ groups by two pyridine type nitrogen atoms $(-\mathrm{N}=)$ at position 3 and $4 .{ }^{3}$ Oxadiazole is a very weak base due to the inductive effect of the extra heteroatom. ${ }^{4}$ The replacement of $(-\mathrm{CH}=)$ groups in furan by two pyridine type nitrogen $(-\mathrm{N}=)$ reduces aromaticity of the resulting oxadiazole ring to such an extent that the oxadiazole ring exhibits the character of conjugated diene. ${ }^{5}$ Due to relatively low electron density on the carbon atom, the oxadiazole ring is extremely resistant towards electrophillic substitutions at carbon atom; however the attack of electrophile occurs at nitrogen, if oxadiazole ring is substituted with electron releasing groups. Nucleo-philic attack is quite difficult in oxadiazole ring; however, halogen substituted oxadiazoles can undergo nucleophilic substitution with replacement of halogen atom by nucleophiles. ${ }^{6}$ These derivative compounds have been found to exhibit diverse biological activities such as analgesic, ${ }^{7}$ anti-inflammatory, ${ }^{8}$ antimicrobial, ${ }^{9}$ anti-HIV,${ }^{10}$ antimalarial, ${ }^{11}$ antifungicidal, ${ }^{12}$ and other biological properties. Some 1,3,4-oxadiazole derivatives have also been applied in the fields of photosensitizers, ${ }^{13}$ liquid crystals, ${ }^{14}$ and organic light-emitting diodes (OLED) $\cdot{ }^{15}$ Consequently, the synthesis of compounds containing this heterocyclic core has attracted considerable attention, and a wide variety of methods has been used for their assembly. The most common synthetic protocol toward the preparation of these compounds involves the dehydrative cyclization of diacylhydrazides using usually strong acidic reagents such as thionyl chloride, ${ }^{16}$ phosphorus pentoxide, ${ }^{17}$ phosphorus oxychloride, ${ }^{18}$ and sulfuric acid. ${ }^{19}$

Literature survey showed that slight modifications in the structure of 1,3,4-oxadiazole can result in quantitative as well as qualitative variations in the biological activity. ${ }^{20}$ So, in the present study we have synthesized various tri-heterocyclic benzamides through a multi-step process to incorporate multi-functionalities in their skeleton. Then, cytotoxicity of these molecules was profiled through hemolytic study on the membrane of red blood cells.

\section{Experimental}

\section{Chemistry}

All the chemicals, along with analytical grade solvents, were purchased from Sigma Aldrich, Alfa Aesar (Germany), or Merck through local suppliers. Pre-coated silica gel Al-plates were used for TLC with ethyl acetate and $n$-hexane as solvent system. Spots were detected by $\mathrm{UV}_{254}$. Gallonkamp apparatus was used to detect melting points in capillary tubes. IR spectra $\left(v_{\text {max }}, \mathrm{cm}^{-1}\right)$ were recorded by $\mathrm{KBr}$ pellet method in the Jasco-320-A spectrophotometer. ${ }^{1} \mathrm{H}-\mathrm{NMR}$ spectra $(\delta, \mathrm{ppm})$ were recorded at $600 \mathrm{MHz}\left({ }^{13} \mathrm{C}-\mathrm{NMR}\right.$ spectra, at $\left.150 \mathrm{MHz}\right)$ in $\mathrm{CDCl}_{3}$ using the Bruker Advance III 600 As- cend spectrometer using $\mathrm{BBO}$ probe. EI-MS spectra were measured on a JEOL JMS$600 \mathrm{H}$ instrument with data processing system. 


\section{Procedure for the preparation of $\{4-[4-($ chloromethyl) benzoyl]- I-piperazinyl\}(2-furyl)methanone (3)}

2-Furyl(1-piperazinyl)methanone $(12.8 \mathrm{mmol} ; 1)$ was taken in an iodine flask $(250 \mathrm{~mL})$ containing $15 \mathrm{~mL}$ of distilled water and $10 \%$ $\mathrm{Na}_{2} \mathrm{CO}_{3}$ (sodium carbonate) solution to adjust $\mathrm{pH}$ at 9-10. Then equimolar 4-(chloromethyl)benzoyl chloride (2) was added dropwise to the reaction medium in 2-5 min. After complete addition, the iodine flask was vigorously shaken (manually) and then set to stir at room temperature for $4 \mathrm{~h}$ till the formation of solid precipitates. The progress of reaction was monitored by thin layer chromatography (TLC) till single spot. The obtained precipitates were filtered, washed with distilled water and dried to yield the titled electrophiles..$^{20,21}$

\section{Procedure for the preparation of ethyl substituted- benzoates (5a-g)}

Substituted-benzoic acids (50mmol; (4a-g, one in each reaction) were taken into a $250 \mathrm{~mL}$ round bottom flasks with a reflux condenser, then absolute ethanol $(40 \mathrm{~mL})$ and conc. sulphuric acid $(1 / 2 \mathrm{~mL})$ were added into the flask and the reaction mixture was refluxed for 3-4h. After maximal completion by thin layer chromatography (TLC), excess water was added and $\mathrm{pH}$ was adjusted to $8-10$ by aqueous solution of sodium carbonate $\left(\mathrm{Na}_{2} \mathrm{CO}_{3} ; 10 \%\right)$. The title compounds were extracted by chloroform.

\section{Procedure for the preparation of substituted- benzohydrazides (6a-g)}

Esters, 5a-g, (40mmol) and $\mathrm{N}_{2} \mathrm{H}_{4} \cdot \mathrm{H}_{2} \mathrm{O}$ (hydrazine monohydrate; $40 \mathrm{mmol}$ ) were taken in a $100 \mathrm{~mL}$ round bottom flask with a reflux condenser and were refluxed for $4-6 \mathrm{~h}$ with $25 \mathrm{~mL}$ methanol. After final thin layer chromatography (TLC), excess water was added to acquire precipitates which were separated by filtration.

\section{Procedure for the preparation of 5-(substituted- phenyl)-I,3,4-oxadiazol-2-thiols (7a-g)}

Acid hydrazides, $6 \mathrm{a}-\mathrm{g}$, (30 mmol) were refluxed for $1 / 2 \mathrm{~h}$ with solid $\mathrm{KOH}$ (potassium hydroxide; $30 \mathrm{mmol}$ ) in $45 \mathrm{~mL}$ ethanol in a $100 \mathrm{~mL}$ round bottom flask. Then $\mathrm{CS}_{2}$ (carbon disulfide; 60mmol) was added and further refluxed for 3-6 h. After final thin layer chromatography (TLC), excess water was added followed by conc. $\mathrm{HCl}$ to adjust $\mathrm{pH}$ of 2 . The mixture was left for $3 \mathrm{~h}$ for precipitation. Precipitates were collected through filtration and washed with water.

General procedure for the preparation of \{4-[4-(\{[5-(substituted-phenyl)-I,3,4-oxadiazol-2yl]sulfanyl\}methyl)benzoyl]- I-piperazinyl\}(2-furyl) methanone (8a-g)

5-(Substituted-phenyl)-1,3,4-oxadiazol-2-thiols (24mmol; 7a-g, one in each reaction) were dissolved in acetonitrile $(20-30 \mathrm{~mL})$ in $100 \mathrm{~mL}$ round bottom flask. Then solid $\mathrm{K}_{2} \mathrm{CO}_{3}$ (potassium carbonate; $12 \mathrm{mmol}$ ) was added. The mixture was refluxed for $1 / 2 \mathrm{~h}$ and then the equimolar $(24 \mathrm{mmol})$ desired electrophile, 3 were added. The mixture was further refluxed for 4-5 hours. Thin layer chromatography (TLC) was carried out to check the reaction completion. Distilled water was added to the reaction mixture to acquire the precipitates. Precipitates were filtered, washed and dried to get the titled compounds. ${ }^{8,17}$
\{4-[4-(\{[5-(2-Chlorophenyl)-I,3,4-oxadiazol-2-yI] sulfanyl\}methyl)benzoyl]-I-piperazinyl\}(2-furyl) methanone (8a)

Light brown liquid; yield: 85\%; Molecular formula: $\mathrm{C}_{25} \mathrm{H}_{21} \mathrm{ClN}_{4} \mathrm{O}_{4} \mathrm{~S}$; molecular mass: 508g/mol; IR (KBr, $\left.v_{\max } \mathrm{cm}^{-1}\right): 3415(\mathrm{~N}-\mathrm{H}), 3063(\mathrm{Ar}$ $\mathrm{C}-\mathrm{H}), 2872(\mathrm{C}-\mathrm{H}), 1654(\mathrm{C}=\mathrm{O}), 1576(\mathrm{C}=\mathrm{C}), 1200(\mathrm{C}-\mathrm{O}-\mathrm{C}), 1112$ (C-N-C), 644 (C-S); ${ }^{~} \mathrm{H}-\mathrm{NMR}\left(600 \mathrm{MHz}, \mathrm{CDCl}_{3}, \delta\right.$ in ppm): $\delta 7.93$ (d, J = 6.0 Hz, 2H, H-2" \& H-6"), 7.55 (d, J = 6.6 Hz, 2H, H-3" \& H-5") 7.52 (br s, 1H, H-5), 7.42-7.39 (m, 4H, H-3"'”, H-4"'”, H-5"', \& H-6"',), 7.07 (d, J = 3.4 Hz, 1H, H-3), 6.50 (dd, J = 1.7, $3.5 \mathrm{~Hz}$, 1H, H-4), 4.54 (s, 2H, H-8"), 3.82 (br s, 4H, H-3' \& H-5'), 3.46 (br s, $4 \mathrm{H}, \mathrm{H}-2$ ' \& H-6'); ${ }^{13} \mathrm{C}-\mathrm{NMR}$ (150 MHz, CDCl3, $\delta$ in ppm): 170.09 (C-7"), 164.33 (C-5"'), 164.15 (C-2"”), 159.2 (C-6), 147.65 (C-2), 143.96 (C-5), 137.96 (C-4”), 134.95 (C-1"'”), 133.03 (C-1”), 132.45 (C-6"'), 131.3 (C-4"”'), 130.92 (C-2"”'), 129.48 (C-5"'), 127.66 (C-3" \& C-5"), 127.11 (C-3"”"), 122.81 (C-2" \& C-6"), 117.26 (C-3), 111.54 (C-4), 52.00 (C-2', C-3', C-5' \& C-6'), 36.27 (C-8'); EI-MS (m/z): $383[\mathrm{M}]+, 259\left[\mathrm{C}_{16} \mathrm{H}_{25} \mathrm{~N}_{2} \mathrm{O}\right]+, 261\left[\mathrm{C}_{15} \mathrm{H}_{21} \mathrm{~N}_{2} \mathrm{O}_{2}\right]+, 204$ $\left[\mathrm{C}_{13} \mathrm{H}_{18} \mathrm{NO}\right]+, 193\left[\mathrm{C}_{10} \mathrm{H}_{13} \mathrm{~N}_{2} \mathrm{O}_{2}\right]+, 150\left[\mathrm{C}_{9} \mathrm{H}_{12} \mathrm{NO}\right]+, 134\left[\mathrm{C}_{8} \mathrm{H}_{8} \mathrm{NO}\right]+$, $107\left[\mathrm{C}_{7} \mathrm{H}_{9} \mathrm{~N}\right] \bullet+, 92\left[\mathrm{C}_{7} \mathrm{H}_{8}\right] \bullet+, 95\left[\mathrm{C}_{5} \mathrm{H}_{3} \mathrm{O}_{2}\right]+$.

\{4-[4-(\{[5-(3-aminophenyl)-I,3,4-oxadiazol-2-yl] sulfanyl\}methyl)benzoyl]- I-piperazinyl\}(2-furyl) methanone (8b)

Light brown liquid; yield: $81 \%$; Molecular formula: $\mathrm{C}_{25} \mathrm{H}_{23} \mathrm{~N}_{5} \mathrm{O}_{4} \mathrm{~S}$; molecular mass: 489g/mol; IR (KBr, vmax cm$\left.~^{-1}\right): 3413(\mathrm{~N}-\mathrm{H}), 3071$ (Ar C-H), 2886 (R C-H), 1658 (C=O), $1580(\mathrm{Ar} \mathrm{C}=\mathrm{C}), 1203$ (C-O-C), 1106 (C-N-C), 653 (C-S); ${ }^{1} \mathrm{H}-\mathrm{NMR}\left(600 \mathrm{MHz}, \mathrm{CDCl}_{3}, \delta\right.$ in ppm): $\delta$ 7.96 (d, J = 7.9 Hz, 1H, H-4"”'), 7.59 (s, 1H, H-2"”'), 7.53-7.49 (m, 2H, H-2" \& H-6"), 7.50 (d, J = 8.1 Hz, 1H, H-6"”), 7.49 (br s, 1H, H-5), 7.42 (t, J = 7.6 Hz, 1H, H-5"'”), 7.30 (d, J = 7.8 Hz, 2H, H-3" \& H-5"), 7.07 (d, J = 2.0 Hz, 1H, H-3), 6.50 (dd, J = 1.6, 3.4 Hz, 1H, H-4), 4.64 (s, 2H, H-8”), 3.85 (br s, 4H, H-3' \& H-5'), 3.52 (br s, $4 \mathrm{H}, \mathrm{H}-2$ ' \& H-6'); ${ }^{13} \mathrm{C}-\mathrm{NMR}\left(150 \mathrm{MHz}, \mathrm{CDCl}_{3}, \delta\right.$ in ppm): 170.09 (C-7”), 164.97 (C-2"'), 164.49 (C-5"'), 159.23 (C-6), 147.63 (C-2), 143.81 (C-5), 137.28 (C-4"), 133.56 (C-1"), 132.06 (C-3"'”), 131.7 (C-1"”'), 130.38 (C-2"”'), 129.29 (C-4"”'), 128.15 (C-5"'”), 127.88 (C-3" \& C-5"), 126.92 (C-6"”'), 122.8 (C-2" \& C-6"), 117.56 (C3), 111.87 (C-4), 45.50 (C-2', C-3', C-5' \& C-6'), 36.76 (C-8'); EIMS (m/z): $489[\mathrm{M}]+, 421\left[\mathrm{C}_{21} \mathrm{H}_{19} \mathrm{~N}_{5} \mathrm{O}_{3} \mathrm{~S}\right] \bullet+, 392\left[\mathrm{C}_{20} \mathrm{H}_{18} \mathrm{~N}_{5} \mathrm{O}{ }_{2} \mathrm{~S}\right]+$, $310\left[\mathrm{C}_{16} \mathrm{H}_{12} \mathrm{~N}_{3} \mathrm{O}_{2} \mathrm{~S}\right]+, 246\left[\mathrm{C}_{13} \mathrm{H}_{14} \mathrm{~N}_{2} \mathrm{O}_{3}\right] \cdot+, 243\left[\mathrm{C}_{12} \mathrm{H}_{9} \mathrm{~N}_{3} \mathrm{OS}\right] \cdot+, 179$ $\left[\mathrm{C}_{9} \mathrm{H}_{11} \mathrm{~N}_{2} \mathrm{O}_{2}\right]+, 161\left[\mathrm{C}_{8} \mathrm{H}_{7} \mathrm{~N}_{3} \mathrm{O}\right]+, 151\left[\mathrm{C}_{8} \mathrm{H}_{9} \mathrm{NO}_{2}\right]+, 95\left[\mathrm{C}_{5} \mathrm{H}_{3} \mathrm{O}_{2}\right]+$.

6\{4-[4-(\{[5-(3-nitrophenyl)-I,3,4-oxadiazol-2-yl] sulfanyl\}methyl)benzoyl]-I-piperazinyl\}(2-furyl) methanone (8c)

Brick red liquid; yield: $82 \%$; Molecular formula: $\mathrm{C}_{25} \mathrm{H}_{21} \mathrm{~N}_{5} \mathrm{O}_{6} \mathrm{~S}$; molecular mass: $519 \mathrm{~g} / \mathrm{mol}$; IR $\left(\mathrm{KBr}, v_{\max } \mathrm{cm}^{-1}\right): 3414(\mathrm{~N}-\mathrm{H}), 3087$ (Ar C-H), $2870(\mathrm{R} \mathrm{C}-\mathrm{H}), 1665(\mathrm{C}=\mathrm{O}), 1579(\mathrm{Ar} \mathrm{C}=\mathrm{C}), 1190$ (C-O-C), 1114 (C-N-C). 645 (C-S); ${ }^{1} \mathrm{H}-\mathrm{NMR}\left(600 \mathrm{MHz}, \mathrm{CDCl}_{3}, \delta\right.$ in ppm): $\delta$ 7.53-7.49 (m, 2H, H-2" \& H-6"), 7.49 (br s, 1H, H-5), 7.44 (s, 1H, H-2"'”), 7.41-7.39 (m, 3H, H-4"'”, H-5"'” \& H-6"'"), 7.30 (d, J = 7.8 Hz, 2H, H-3" \& H-5") 7.07 (d, J = 2.0 Hz, 1H, H-3), 6.50 (dd, J = 1.6, $3.4 \mathrm{~Hz}, 1 \mathrm{H}, \mathrm{H}-4), 4.64$ (s, 2H, H-8"), 3.85 (br s, 4H, H-3' \& H-5'), 3.52 (br s, $4 \mathrm{H}, \mathrm{H}-2$ ' \& H-6'); ${ }^{13} \mathrm{C}-\mathrm{NMR}\left(150 \mathrm{MHz}, \mathrm{CDCl}_{3}, \delta\right.$ in ppm): 170.05 (C-7”), 164.34 (C-5"'), 164.17 (C-2"'), 159.23 (C-6), 149.53 (C-3"'”), 147.6 (C-2), 143.91 (C-5), 137.99 (C-4"), 133.09 (C-1"), 130.78 (C-6"'”), 129.23 (C-5"'”), 128.48 (C-1"'”), 127.66 (C- 
3" \& C-5”), 126.04 (C-4"”'), 125.4 (C-2"”'), 122.8 (C-2" \& C-6"), 117.26 (C-3), 111.55 (C-4), 48.05 (C-2', C-3', C-5' \& C-6'), 36.23 (C-8"); EI-MS (m/z): $397[\mathrm{M}]+, 273\left[\mathrm{C}_{17} \mathrm{H}_{25} \mathrm{~N}_{2} \mathrm{O}\right]+, 261\left[\mathrm{C}_{15} \mathrm{H}_{21} \mathrm{~N}_{2} \mathrm{O}_{2}\right]$ $+, 218\left[\mathrm{C}_{14} \mathrm{H}_{20} \mathrm{NO}\right]+, 193\left[\mathrm{C}_{10} \mathrm{H}_{13} \mathrm{~N}_{2} \mathrm{O}_{2}\right]+, 164\left[\mathrm{C}_{10} \mathrm{H}_{14} \mathrm{NO}\right]+, 148$ $\left[\mathrm{C}_{9} \mathrm{H}_{10} \mathrm{NO}\right]+, 121\left[\mathrm{C}_{8} \mathrm{H}_{11} \mathrm{~N}\right] \bullet+, 106\left[\mathrm{C}_{8} \mathrm{H}_{10}\right] \bullet+, 95\left[\mathrm{C}_{5} \mathrm{H}_{3} \mathrm{O}_{2}\right]+$.

\{4-[4-(\{[5-(4-methylphenyI)-I,3,4-oxadiazol-2-yI] sulfanyl\}methyl)benzoyl]- I-piperazinyl\}(2-furyl) methanone (8d)

Light brown liquid; yield: $88 \%$; Molecular formula: $\mathrm{C}_{26} \mathrm{H}_{24} \mathrm{~N}_{4} \mathrm{O}_{4} \mathrm{~S}$; molecular mass: 488g/mol; IR (KBr, vmax cm$\left.{ }^{-1}\right): 3410(\mathrm{~N}-\mathrm{H}), 3088$ (Ar C-H), $2879(\mathrm{C}-\mathrm{H}), 1661(\mathrm{C}=\mathrm{O}), 1578(\mathrm{C}=\mathrm{C}), 1199(\mathrm{C}-\mathrm{O}-\mathrm{C}), 1111$ (C-N-C), 657 (C-S); ${ }^{1} \mathrm{H}-\mathrm{NMR}\left(600 \mathrm{MHz}, \mathrm{CDCl}_{3}, \delta\right.$ in ppm): $\delta 7.87$ (d, $\mathrm{J}=6.3 \mathrm{~Hz}, 2 \mathrm{H}, \mathrm{H}-2$ " \& H-6"), 7.55 (d, J = 6.4 Hz, 2H, H-3" \& H-5") 7.46 (br s, 1H, H-5), 7.40 (d, J = 6.4 Hz, 2H, H-2"'” \& H-6"'”), 7.29 (d, J = 6.3 Hz, 2H, H-3"'” \& H-5"'”), 7.07-7.05 (m, 1H, H-3), 6.516.49 (m, 1H, H-4), 4.52 (s, 2H, H-8'), 3.9 (br s, 4H, H-3' \& H-5'), 3.5 (br s, 4H, H-2' \& H-6'), 2.42 (s, 3H, H-7'"')); ${ }^{13} \mathrm{C}-\mathrm{NMR}(150 \mathrm{MHz}$, $\mathrm{CDCl3}, \delta$ in ppm): 170.09 (C-7"), 166.2 (C-5"'), 163.04 (C-2"'), 159.2 (C-6), 147.64 (C-2), 143.96 (C-5), 142.39 (C-4"), 138.13 (C1"), 134.89 (C-4"”'), 129.79 (C-3" \& C-5"), 128.83 (C-2" \& C-6"), 127.64 (C-2"'” \& C-6"'”), 126.64 (C-3"'” \& C-5"'”), 120.74 (C-1"'”), 117.28 (C-3), 111.55 (C-4), 45.45 (C-2', C-3', C-5' \& C-6'), 36.28 (C-8"), 21.64 (C-7"'"); EI-MS (m/z): 488 [M]+, $273\left[\mathrm{C}_{17} \mathrm{H}_{25} \mathrm{~N}_{2} \mathrm{O}\right]+$, $261\left[\mathrm{C}_{15} \mathrm{H}_{21} \mathrm{~N}_{2} \mathrm{O}_{2}\right]+, 218\left[\mathrm{C}_{14} \mathrm{H}_{20} \mathrm{NO}\right]+, 193\left[\mathrm{C}_{10} \mathrm{H}_{13} \mathrm{~N}_{2} \mathrm{O}_{2}\right]+, 164$ $\left[\mathrm{C}_{10} \mathrm{H}_{14} \mathrm{NO}\right]+, 148\left[\mathrm{C}_{9} \mathrm{H}_{10} \mathrm{NO}\right]+, 121\left[\mathrm{C}_{8} \mathrm{H}_{11} \mathrm{~N}\right] \bullet+, 106\left[\mathrm{C}_{8} \mathrm{H}_{10}\right] \bullet+, 95$ $\left[\mathrm{C}_{5} \mathrm{H}_{3} \mathrm{O}_{2}\right]+$.

\{4-[4-(\{[5-(4-hydroxyphenyl)-I,3,4-oxadiazol-2-yl] sulfanyl\}methyl)benzoyl]- I-piperazinyl\}(2-furyl) methanone (8e)

Black brown liquid; yield: $84 \%$; Molecular formula: $\mathrm{C}_{25} \mathrm{H}_{22} \mathrm{~N}_{4} \mathrm{O}_{5} \mathrm{~S}$; molecular mass: 490g/mol; IR (KBr, $\left.v_{\max } \mathrm{cm}^{-1}\right): 3416(\mathrm{~N}-\mathrm{H}), 3089$ (Ar C-H), $2870(\mathrm{C}-\mathrm{H}), 1661(\mathrm{C}=\mathrm{O}), 1574(\mathrm{C}=\mathrm{C}), 1191(\mathrm{C}-\mathrm{O}-\mathrm{C})$, $1116(\mathrm{C}-\mathrm{N}-\mathrm{C}), 649$ (C-S); ${ }^{1} \mathrm{H}-\mathrm{NMR}\left(600 \mathrm{MHz}, \mathrm{CDCl}_{3}, \delta\right.$ in ppm): $\delta 7.93$ (d, J = 9.0 Hz, 2H, H-2"" \& \& H-6""'), 7.80 (d, J = $6.9 \mathrm{~Hz}$, 2H, H-2" \& H-6"), 7.56 (d, J = $7.5 \mathrm{~Hz}, 2 \mathrm{H}, \mathrm{H}-3$ " \& H-5") 7.51 (d, J = 1.9 Hz, 1H, H-5), 7.09 (d, J = 3.7 Hz, 1H, H-3), 6.92-6.90 (d, J = 6.3 Hz, 2H, H-3"'” \& H-5"'”), 6.51 (dd, J = 1.9, 4.3 Hz, 1H, H-4), 4.52 (s, 2H, H-8"), 3.9 (br s, 4H, H-3' \& H-5'), 3.56 (br s, 4H, $\mathrm{H}-2$ ' \& H-6'); ${ }^{13} \mathrm{C}-\mathrm{NMR}\left(150 \mathrm{MHz}, \mathrm{CDCl}_{3}, \delta\right.$ in ppm): 170.48 (C7"'), 166.22 (C-5"'), 162.40 (C-2"”), 160.09 (C-4"”'), 159.27 (C-6), 147.53 (C-2), 144.07 (C-5), 140.29 (C-4"), 138.30 (C-1"), 130.03 (C-3" \& C-5"), 129.12 (C-1"”'), 127.31 (C-2" \& C-6"), 117.45 (C3), 116.31 (C-2"'” \& C-6"'”), 115.40 (C-3"'” \& C-5"'”), 111.59 (C4), 52.24 (C-2', C-3', C-5' \& C-6'), 36.35 (C-8'); EI-MS (m/z): 397 $[\mathrm{M}]+, 273\left[\mathrm{C}_{17} \mathrm{H}_{25} \mathrm{~N}_{2} \mathrm{O}\right]+, 261\left[\mathrm{C}_{15} \mathrm{H}_{21} \mathrm{~N}_{2} \mathrm{O}_{2}\right]+, 218\left[\mathrm{C}_{14} \mathrm{H}_{20} \mathrm{NO}\right]+, 193$ $\left[\mathrm{C}_{10} \mathrm{H}_{13} \mathrm{~N}_{2} \mathrm{O}_{2}\right]+, 164\left[\mathrm{C}_{10} \mathrm{H}_{14} \mathrm{NO}\right]+, 148\left[\mathrm{C}_{9} \mathrm{H}_{10} \mathrm{NO}\right]+, 121\left[\mathrm{C}_{8} \mathrm{H}_{11} \mathrm{~N}\right] \bullet+$, $106\left[\mathrm{C}_{8} \mathrm{H}_{10}\right] \bullet+, 95\left[\mathrm{C}_{5} \mathrm{H}_{3} \mathrm{O}_{2}\right]+$.

\{4-[4-(\{[5-(2,4-dichlorophenyl)-I,3,4-oxadiazol-2yl] sulfanyl\}methyl)benzoyl]- I-piperazinyl\}(2-furyl) methanone (8f)

Light brown liquid; yield: $80 \%$; Molecular formula: $\mathrm{C}_{25} \mathrm{H}_{20} \mathrm{ClN}_{4} \mathrm{O}_{4} \mathrm{~S}$; molecular mass: 542g/mol; IR $\left(\mathrm{KBr}, v_{\max } \mathrm{cm}^{-1}\right): 3405$ $(\mathrm{N}-\mathrm{H}), 3082$ (Ar C-H), $2880(\mathrm{R} \mathrm{C}-\mathrm{H}), 1655(\mathrm{C}=\mathrm{O}), 1583(\mathrm{Ar} \mathrm{C}=\mathrm{C})$, 1199 (C-O-C), 1110 (C-N-C), 644 (C-S); ${ }^{1} \mathrm{H}-\mathrm{NMR}\left(600 \mathrm{MHz}, \mathrm{CDCl}_{3}\right.$, $\delta$ in ppm): $\delta 7.89$ (d, J = 8.5 Hz, 2H, H-2" \& H-6"), 7.56-7.55 (m, 2H, H-5"' \& H-6"'”), 7.54 (s, 1H, H-3"'”), 7.41 (d, J = 6.3 Hz, 2H, H-3"
\& H-5") 7.49 (br s, 1H, H-5), 7.06 (d, J = 2.5 Hz, 1H, H-3), 6.50 (dd, $\mathrm{J}=1.7,3.4 \mathrm{~Hz}, 1 \mathrm{H}, \mathrm{H}-4), 4.56$ (s, 2H, H-8"), 3.85 (br s, 4H, H-3' \& H-5'), 3.54 (br s, 4H, H-2' \& H-6'); ${ }^{13} \mathrm{C}-\mathrm{NMR}\left(150 \mathrm{MHz}, \mathrm{CDCl}_{3}, \delta\right.$ in ppm): 170.04 (C-7”), 164.38 (C-5"'), 163.58 (C-2"'), 159.18 (C-6), 147.62 (C-2), 143.96 (C-5), 138.18 (C-4"), 137.85 (C-4"”'), 134.99 (C-1"”'), 133.75 (C-6"'”), 131.55 (C-1"), 131.24 (C-5"'”), 129.58 (C3" \& C-5"), 129.48 (C-2"”"), 127.66 (C-3"”"), 127.45 (C-2" \& C-6"), 117.26 (C-3), 111.54 (C-4), 48.50 (C-2', C-3', C-5' \& C-6'), 36.24 (C8"); EI-MS (m/z): $369[\mathrm{M}]+, 245\left[\mathrm{C}_{15} \mathrm{H}_{21} \mathrm{~N}_{2} \mathrm{O}\right]+, 261\left[\mathrm{C}_{15} \mathrm{H}_{21} \mathrm{~N}_{2} \mathrm{O}_{2}\right]+$, $193\left[\mathrm{C}_{10} \mathrm{H}_{13} \mathrm{~N}_{2} \mathrm{O}_{2}\right]+, 190\left[\mathrm{C}_{12} \mathrm{H}_{16} \mathrm{NO}\right]+,, 136 \quad\left[\mathrm{C}_{8} \mathrm{H}_{10} \mathrm{NO}\right]+, \quad 120$ $\left[\mathrm{C}_{7} \mathrm{H}_{6} \mathrm{NO}\right]+, 93\left[\mathrm{C}_{6} \mathrm{H}_{6} \mathrm{~N}\right] \bullet+, 78\left[\mathrm{C}_{6} \mathrm{H}_{6}\right] \bullet+, 95\left[\mathrm{C}_{5} \mathrm{H}_{3} \mathrm{O}_{2}\right]+$.

\section{$\{4-[4-(\{[5-(3,5-d i n i t r o p h e n y I)-I, 3,4-o x a d i a z o l-2-y \mid]$ sulfanyl\}methyl)benzoyl]- I-piperazinyl\}(2-furyl) methanone (8g)}

Light brown liquid; yield: $84 \%$; Molecular formula: $\mathrm{C}_{25} \mathrm{H}_{20} \mathrm{~N}_{6} \mathrm{O}_{8} \mathrm{~S}$; molecular mass: 564g/mol; IR (KBr, $\left.v_{\max } \mathrm{cm}^{-1}\right): 3415(\mathrm{~N}-\mathrm{H}), 3062$ (Ar C-H), $2880(\mathrm{R} \mathrm{C}-\mathrm{H}), 1653(\mathrm{C}=\mathrm{O}), 1582(\mathrm{Ar} \mathrm{C}=\mathrm{C}), 1205(\mathrm{C}-\mathrm{O}-\mathrm{C})$, 1107 (C-N-C), $658(\mathrm{C}-\mathrm{S}) ;{ }^{1} \mathrm{H}-\mathrm{NMR}\left(600 \mathrm{MHz}, \mathrm{CDCl}_{3}, \delta\right.$ in ppm): $\delta 8.05$ (s, 1H, H-4"'"), $7.58(\mathrm{~d}, \mathrm{~J}=6.3 \mathrm{~Hz}, 2 \mathrm{H}, \mathrm{H}-5$ "” \& H-6" "'), 7.50-7.44 (m, 2H, H-2" \& H-6"), 7.46 (br s, 1H, H-5), 7.27 (d, J = $7.8 \mathrm{~Hz}, 2 \mathrm{H}, \mathrm{H}-3$ " \& H-5") 7.00 (d, J = 2.0 Hz, 1H, H-3), 6.54 (dd, J $=1.6,3.4 \mathrm{~Hz}, 1 \mathrm{H}, \mathrm{H}-4), 4.68$ (s, 2H, H-8"), 3.80 (br s, 4H, H-3' \& $\mathrm{H}-5^{\prime}$ ), 3.57 (br s, 4H, H-2' \& H-6'); ${ }^{13} \mathrm{C}-\mathrm{NMR}\left(150 \mathrm{MHz}, \mathrm{CDCl}_{3}\right.$, $\delta$ in ppm): 170.05 (C-7"), 164.31 (C-5"”), 164.12 (C-2"'), 159.20 (C-6), 150.08 (C-3"'” \& C-5"'”), 147.66 (C-2), 143.94 (C-5), 137.90 (C-4"), 134.24 (C-4"'”), 133.04 (C-1"), 131.46 (C-1"'”), 130.79 (C2"'” \& C-6"”'), 127.69 (C-3" \& C-5"), 122.86 (C-2" \& C-6"), 117.27 (C-3), 111.52 (C-4), 45.48 (C-2', C-3', C-5' \& C-6'), 36.24 (C-8”); EI-MS (m/z): $564[\mathrm{M}]+, 496\left[\mathrm{C}_{21} \mathrm{H}_{16} \mathrm{~N}_{6} \mathrm{O}_{7} \mathrm{~S}\right] \cdot+, 467\left[\mathrm{C}_{20} \mathrm{H}_{15} \mathrm{~N}_{6} \mathrm{O}_{6} \mathrm{~S}\right]+$, $385\left[\mathrm{C}_{16} \mathrm{H}_{9} \mathrm{~N}_{4} \mathrm{O} 6 \mathrm{~S}\right]+, 318\left[\mathrm{C}_{12} \mathrm{H}_{6} \mathrm{~N}_{4} \mathrm{O}_{5} \mathrm{~S}\right] \cdot+, 246\left[\mathrm{C}_{13} \mathrm{H}_{14} \mathrm{~N}_{2} \mathrm{O}_{3}\right] \bullet+, 236$ $\left[\mathrm{C}_{8} \mathrm{H}_{4} \mathrm{~N}_{4} \mathrm{O}_{5}\right]+, 179\left[\mathrm{C}_{9} \mathrm{H}_{11} \mathrm{~N}_{2} \mathrm{O}_{2}\right]+, 151\left[\mathrm{C}_{8} \mathrm{H}_{9} \mathrm{NO}_{2}\right]+, 118\left[\mathrm{C}_{6} \mathrm{H}_{2} \mathrm{~N}_{2} \mathrm{O}\right]+$, $95\left[\mathrm{C}_{5} \mathrm{H}_{3} \mathrm{O}_{2}\right]+$.

\section{Hemolytic activity assay}

Bovine blood sample was collected in Ethylene Diamine Tetra Acetic acid (EDTA) that was diluted with saline $(0.9 \% \mathrm{NaCl})$, and centrifuge at $1000 \mathrm{xg}$ for $10 \mathrm{~min}$. The erythrocytes separated diluted in phosphate buffer saline of $\mathrm{pH} 7.4$ and a suspension was made. Add $20 \mu \mathrm{L}$ of synthetic compounds solution $(10 \mathrm{mg} / \mathrm{mL})$ in $180 \mu \mathrm{L}$ of RBCs suspension and incubate for $30 \mathrm{~min}$ at room temperature. Phosphatebuffered saline (PBS) was used as negative control and Triton 100-X was taken as positive control. ${ }^{21,22}$ The $\%$ age of hemolysis was taken as by using formula:

$(\%)$ of Hemolysis $=\frac{\text { Absorbance of sample }- \text { Absorbance of negative control }}{\text { Absorbance of positive control }} \times 100$

\section{Results and discussion}

\section{Chemistry}

In the present investigation, various novel tri-heterocyclic benzamides were synthesized by the nucleophilic substitution reaction of 2-furoyl-(1-piperazinyl)methanone (1) with 4-(chloromethyl) benzoyl chloride (2) in a basic aqueous medium to get an electrophile \{4-[4-(chloromethyl)benzoyl]-1-piperazinyl\}(2-furyl) methanone (3). In parallel set of reactions, various substituted-benzoic acids (4a-g) were refluxed with ethanol and conc. sulphuric acid to form respective ethyl substituted-benzoates $(5 \mathrm{a}-\mathrm{g})$. These esters were 
further refluxed with $\mathrm{N}_{2} \mathrm{H}_{4} \cdot \mathrm{H}_{2} \mathrm{O}$ in methanol solution to acquire substituted-benzohydrazides (6a-g). These hydrazides were cyclized into heterocyclic core by refluxing with $\mathrm{CS}_{2}$ in the presence of $\mathrm{KOH}$ and ethanol solvent, giving rise to the formation of various 5-(substituted-phenyl)-1,3,4-oxadiazol-2-thiols (7a-g). The final step in the synthesis was the coupling of electrophile, 3 , with nucelophiles, $7 \mathrm{a}-\mathrm{g}$, in acetonitrile and potassium carbonate to yield the targeted triheterocyclic molecules, 8a-g. Structures of these novel compounds were characterized and confirmed by IR, ${ }^{1} \mathrm{H}-\mathrm{NMR},{ }^{13} \mathrm{C}-\mathrm{NMR}$ and EIMS techniques. (Figure 1) (Table 1).

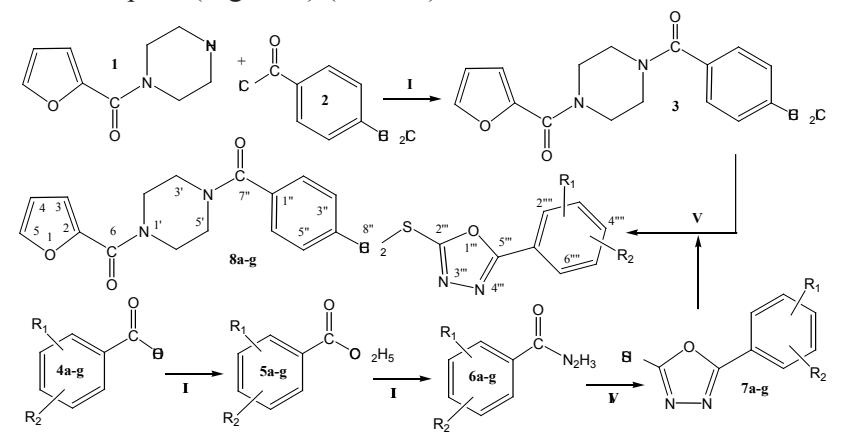

Figure I Outline for the synthesis of novel tri-heterocyclic benzamides. Reagents \& Conditions:

a. Aq. $\mathrm{Na}_{2} \mathrm{CO}_{3}$ soln./pH 9-10/stirring at RT for $4 \mathrm{hrs}$.

b. $\mathrm{EtOH} / \mathrm{H}_{2} \mathrm{SO}_{4} /$ refluxing for 3-4 hrs.

c. $\mathrm{MeOH} / \mathrm{N}_{2} \mathrm{H}_{4} \cdot \mathrm{H}_{2} \mathrm{O} /$ refluxing for 4-6 hrs.

d. $\mathrm{EtOH} / \mathrm{CS}_{2} / \mathrm{KOH} /$ refluxing for 3-6 hrs.

e. Acetonitrile/ $/ \mathrm{K}_{2} \mathrm{CO}_{3} /$ refluxing for $0.5 \mathrm{hrs}$ for activation of $7 \mathrm{a}-\mathrm{g}$ (one in each reaction), followed by addition of 3 and finally refluxing for 4-5 hrs.

Table I List of -RI and -R2 substituents in novel tri-heterocyclic benzamides (8a-g)

\begin{tabular}{lll}
\hline Compound & $\mathbf{R}_{\mathbf{1}}$ & $\mathbf{R}_{\mathbf{2}}$ \\
\hline $8 \mathrm{a}$ & $2-\mathrm{Cl}$ & $\mathrm{H}$ \\
$8 \mathrm{~b}$ & $3-\mathrm{NH}_{2}$ & $\mathrm{H}$ \\
$8 \mathrm{c}$ & $3-\mathrm{NO}_{2}$ & $\mathrm{H}$ \\
$8 \mathrm{~d}$ & $4-\mathrm{CH}_{3}$ & $\mathrm{H}$ \\
$8 \mathrm{e}$ & $4-\mathrm{OH}$ & $\mathrm{H}$ \\
$8 \mathrm{f}$ & $2-\mathrm{Cl}$ & $4-\mathrm{Cl}$ \\
$8 \mathrm{~g}$ & $3-\mathrm{NO}_{2}$ & $5-\mathrm{NO}_{2}$ \\
\hline
\end{tabular}

One of the compounds is discussed hereby in detail for the expediency of the readers. For example, compound 8d, IR absorption band of aromatic $\mathrm{C}-\mathrm{H}$ str. appeared at 3088, aliphatic $\mathrm{C}-\mathrm{H}$ str. at 2879, $1661(\mathrm{C}=\mathrm{O}), 1578$ (Ar C=C), 1199 (C-O-C), 1111 (C-N-C), 657 (C-S). Its molecular formula was confirmed through EI-MS showing molecular ion peak at $\mathrm{m} / \mathrm{z} 192,119,95$ corresponding to $\mathrm{C}_{26} \mathrm{H}_{24} \mathrm{~N}_{4} \mathrm{O}_{4} \mathrm{~S}$ (Calcd. for 488). The EI-MS spectral data was also in complete agreement for the proposed structure of $8 \mathrm{~d}$ which was finally confirmed through its ${ }^{1} \mathrm{H}-\mathrm{NMR}$ and ${ }^{13} \mathrm{C}-\mathrm{NMR}$ spectra.

In ${ }^{1} \mathrm{H}-\mathrm{NMR}$ spectrum, signals of methylbenzamide moiety appeared at $\delta 7.87$ (d, J = 6.3 Hz, 2H, H-2", H-6”), 7.55 (d, J = 6.4 $\mathrm{Hz}, 2 \mathrm{H}, \mathrm{H}-3$ ", H-5") and 4.52 (s, 2H, H-8"). The signals of protons for 4-methylphenyl ring appeared at $7.40(\mathrm{~d}, \mathrm{~J}=6.4 \mathrm{~Hz}, 2 \mathrm{H}, \mathrm{H}-2$ "', H-6"'”), 7.29 (d, J = 6.3 Hz, 2H, H-3"'”, H-5"'”) and 2.42 (s, 3H,
CH3-7"')' Furan ring showed three peaks in aromatic region at $\delta$ 7.46 (br.s, $1 \mathrm{H}, \mathrm{H}-5), 7.07-7.05(\mathrm{~m}, 1 \mathrm{H}, \mathrm{H}-3)$ and 6.51-6.49 (m, $1 \mathrm{H}$, $\mathrm{H}-4$ ). The eight protons of piperazine ring appeared at $\delta 3.90$ (br.s, $4 \mathrm{H}$, CH2-3', CH2-5') and 3.50 (br.s, 4H, CH2-2', CH2-6').

The structure was also thorough supported by its ${ }^{13} \mathrm{C}-\mathrm{NMR}$ spectrum. Six signals of 170.09 (C-7"), 142.39 (C-4"), 138.13 (C1"), 129.79 (C-3", C-5"), 128.83 (C-2", C-6") and 36.28 (C-8") supported the 4-methylenebenzamide. Two signals of 166.20 (C-5"') and 163.04 (C-2"') corroborated the 1,3,4-oxadiazole ring present in the molecule. Five signals of 159.20 (C-6), 147.64 (C-2), 143.96 (C5), $117.28(\mathrm{C}-3)$ and $111.55(\mathrm{C}-4)$ confirmed the furoyl. Five signals of 134.89 (C-6"”'), 127.64 (C-2"', C-6"”'), 126.64 (C-3"', C-5"”'), 120.74 (C-1"'”) and 21.64 (C-7"'”) confirmed the 4-methylphenyl ring. Piperazine was corroborated by single signal of 45.45 (C-2', C-3', C-5', C-6'). So, on the basis of above discussed cumulative evidences, the structure of $8 \mathrm{~d}$ was named as $\{4-[4-(\{[5-(4-M e t h y l p h e n y l)-$ 1,3,4-oxadiazol-2-yl] sulfanyl $\}$ methyl)benzoyl]-1-piperazinyl $\}$ (2-furyl)methanone. Similarly all other synthesized derivatives were characterized by aforesaid spectral techniques.

\section{The \%age hemolytic activity and structure-activity relationship (8a-g)}

All the synthesized compounds were subjected to hemolytic assay to find out their cytotoxicity profile. Results of percentage hemolysis are shown in Table 2 indicate that all the compounds are nearly nontoxic for membrane of red blood cells. Maximum membrane toxicity was seen by the compound $8 \mathrm{e}(10.90 \%)$ due to hydroxyl group substitution at para position while minimum was noted in compounds 8 a $(0.76 \%)$ in which at the $o$-position occupied by a chloryl group. Overall very mild toxicity was observed for molecules $8 \mathrm{~d}(2.98 \%), 8 \mathrm{c}$ (4.21\%), $8 \mathrm{~g}(4.25 \%), 8 \mathrm{~b}(7.94 \%)$ and $8 \mathrm{f}(10.77 \%)$ relative to PBS and Triton-X having $\%$ hemolysis of $0.09 \%$ and $100 \%$ respectively.

Table 2 Hemolytic activity of synthesized compounds, $\{4-[4-(\{[5-($ substituted)I,3,4-oxadiazol-2-yl]sulfanyl\}methyl)benzoyl]-I-piperazinyl\}(2-furyl) methanone (8a-g).

\begin{tabular}{ll}
\hline Compounds & Hemolytic Activity (\%) \\
\hline $8 \mathrm{a}$ & 0.76 \\
$8 \mathrm{~b}$ & 7.94 \\
$8 \mathrm{c}$ & 4.21 \\
$8 \mathrm{~d}$ & 2.98 \\
$8 \mathrm{e}$ & 10.9 \\
$8 \mathrm{f}$ & 10.77 \\
$8 \mathrm{~g}$ & 4.25 \\
Triton-X-100 & 100 \\
PBS & 0.09 \\
\hline
\end{tabular}

\section{Conclusion}

The anticipated structures of the synthesized tri-heterocyclic molecules, 8a-g, were thoroughly supported by spectroscopic analysis. The hemolytic activity data of these molecules revealed that these have low cytotoxicity and hence might be considered as safe therapeutic agents in drug discovery program. 


\section{Acknowledgements}

The Higher Education Commission (HEC) of Pakistan is highly acknowledged by the authors for financial support regarding purchasing of chemicals and spectral study.

\section{Conflict of interest}

There is no conflict of interest.

\section{References}

1. Sengupta P, Mal M, Mandal S, et al. Evaluation of Antibacterial and Antifungal Activity of some 1, 3, 4 Oxadiazoles. Iranian J Pharm Therap. 2008;7(2):165-167.

2. Kadi AA, El-Brollosy NR, Al-Deeb OA, et al. Synthesis, antimicrobial, and anti-inflammatory activities of novel 2-(1-adamantyl)-5-substituted1,3,4-oxadiazoles and 2-(1-adamantylamino)-5-substituted-1,3,4thiadiazoles. Eur J Med Chem. 2007;42(2):235-242.

3. N Bhardwaj, SK Saraf, P Sharma, et al. Syntheses, Evaluation and Characterization of Some 1, 3, 4-Oxadiazoles as Antimicrobial Agents. $E$ J Chem. 2009;6(4):1133-1138.

4. Sahu VKR, Singh AK, Yadav D. Review article on 1, 3, 4-Oxadiazole derivaties and it's Pharmacological activities. Int $J$ Chem Tech Res. 2011;3(3):1362-1372.

5. Nagaraj, Chaluvaraju KC, Niranjan MS, et al. 1, 3, 4-Oxadiazole: a potent drug candidate with various pharmacological activities. Int $J$ Pharmacy and Pharm Sci. 2011;3(3):9-16.

6. Bhatia S, Gupta M. 1, 3, 4-Oxadiazole as antimicrobial agents: An overview. J Chem Pharm Res. 2011;3(3):137-147.

7. Jayashankar B, Rai KML, Baskaran N, et al. Synthesis and pharmacological evaluation of 1,3,4-oxadiazole bearing bis(heterocycle) derivatives as anti-inflammatory and analgesic agents. Eur J Med Chem. 2009;44(10):3898-3902.

8. Alam MM, Shaharyar M, Hamid H, et al. Synthesis of novel 8-hydroxy quinolin based 1,3,4-oxadiazoles and S-substituted 1,2,4-triazole derivatives and evaluation of their anti-inflammatory, analgesic, ulcerogenic and anti-microbial activities. Med Chem. 2011;7(6):663673.

9. Gupta V, Kashaw SK, Jatav V, et al. Synthesis and antimicrobial activity of some new 3-[5-(4-substituted) phenyl-1,3,4-oxadiazole-2yl]-2styrylquinazoline-4(3H)-ones. Med Chem Res. 2008;17(2-7):205-211.

10. El-Emam AA, Al-Deeb OA, Al-Omar M, et al. Synthesis, antimicrobial, and anti-HIV-1 activity of certain 5-(1-adamantyl)-2-substituted thio1,3,4-oxadiazoles and 5-(1-adamantyl)-3-substituted aminomethyl-1,3,4oxadiazoline-2-thiones. Bioorg Med Chem. 2004;12(19):5107-5113.
11. Zareef M, Rashid I, De Dominguez NG, et al. Synthesis and antimalarial activity of novel chiral and achiral benzenesulfonamides bearing 1, 3, 4-oxadiazole moieties. J Enzym Inhib Med Chem. 2007;22(3):301-308.

12. $\mathrm{Xu} \mathrm{W}, \mathrm{He} \mathrm{J}, \mathrm{He} \mathrm{M}$, et al. Synthesis and antifungal activity of novel sulfone derivatives containing 1,3,4-oxadiazole moieties. Molecules. 2011;16(11):9129-9141.

13. Wang JF, Jabbour GE, Mash EA, et al. Oxadiazole Metal Complex for Organic Light-Emitting Diodes. Adv Mater. 1999;11(15):1266-1269.

14. Hernández-Ainsa S, Barberá J, Marcos $\mathrm{M}$, et al. Liquid Crystalline Ionic Dendrimers Containing Luminescent Oxadiazole Moieties. Macromolecules. 2012;45(2):1006-1015.

15. Linton KE, Fisher AL, Pearson C, et al. Colour tuning of blue electroluminescence using bipolar carbazole-oxadiazole molecules in single-active-layer organic light emitting devices (OLEDs). $J$ Mater Chem. 2012;22(23):11816-11825.

16. Wagner E, Al-Kadasi K, Zimecki M, et al. Synthesis and pharmacological screening of derivatives of isoxazolo[4,5-d]pyrimidine. Eur J Med Chem. 2008;43(11):2498-2504.

17. Sharba AHK, Al-Bayati RH, Aouad M, et al. Synthesis of Oxadiazoles, Thiadiazoles and Triazoles Derived from Benzo[b]thiophene. Molecules. 2005;10(9):1161-1168.

18. Husain A, Rashid M, Mishra R, et al. Benzimidazole bearing oxadiazole and triazolo-thiadiazoles nucleus: design and synthesis as anticancer agents. Bioorg Med Chem Lett. 2012;22(17):5438-5444.

19. Sharma S, Srivastava VK, Kumar A. Newer N-substituted anthranilic acid derivatives as potent anti-inflammatory agents. Eur J Med Chem. 2002;37(8):689-697.

20. Abbasi MA, Akhtar A, Aziz-ur-Rehman, et al. Synthesis, structural characterization and enzyme inhibition studies on 5-(2-nitrostyryl)-1,3,4oxadiazole-2-thiol derivatives. J Chil Chem Soc. 2013;58(4):2186-2190.

21. Sharma P, Sharma JD. In vitro hemolysis of human erythrocytes -by plant extracts with antiplasmodial activity. $J$ Ethnopharmacol. 2001;74(3):239-243.

22. Stepanovic S, Vukovic D, Dakic I, et al. A modified microtiter-plate test for quantification of staphylococcal biofilm formation. $J$ Microbiol Methods. 2000;40(2):175-179. 